\title{
The validation of the sit-to-stand test for COPD patients
}

To the Editor:

We read with interest the publication by Своок et al. [1] on the validation and responsive properties of the 1-min sit-to-stand (STS) test in patients with chronic obstructive pulmonary disease (COPD) undergoing pulmonary rehabilitation. The authors performed a comprehensive evaluation of the minimal clinical meaningful difference of the 1-min STS test. In our own dataset of patients from a multicentre study, this STS test exhibited similar level of reliability, intra-subject repeatability [2], and responsiveness to pulmonary rehabilitation with an estimated minimal important difference (MID) of three repetitions [3]. In their study, Своок et al. [1] emphasised the change in STS repetitions, which is better related to change in subjective outcomes (feeling thermometer notably), rather than with physical capacity outcomes such as the 6-min walk distance (6MWD). It is, after all, largely accepted that health-related quality of life (HRQoL) tools are the most sensitive in pulmonary rehabilitation, given the multimodal and patient-tailored interventions addressed in order to optimise benefits, not only focused on exercise training, but also on change in education and behaviour [4].

However, in the STAND-UP group of their study [1], the absence of correlation between the change in STS repetitions and the $6 \mathrm{MWD}$ on one side, and between the change in STS repetitions and the quadriceps maximal voluntary contraction (QMVC) on the other side remains puzzling (table 3 of the article). We were wondering if this could be attributed to specific component of the STAND-UP pulmonary rehabilitation programme.

Given the correlation observed between the changes in STS repetitions and QMVC ( $\mathrm{r}=0.24$; table 3 of the article), one should expect that raw value of change in STS repetitions and 6MWD be correlated in STAND-UP. This result differs substantially from the result obtained in the RIMTCORE group of their study [1], and our data obtained from a prospective multicentre study including a group of $48 \mathrm{COPD}$ patients [3] with slightly less severe disease in terms of forced expiratory volume in $1 \mathrm{~s} \mathrm{(FEV1)}(52.3 \pm 16.9 \%$ predicted versus $40.6 \pm 14.7 \%$ ). Could this difference be attributed to the heterogeneity of the STAND-UP subjects, drastically reducing the raw correlations between STS repetitions and 6MWD?

In our data, the relationship between STS and QMVC confirms that muscle strength is an important determinant of 1-min STS performance. This test also integrates postural control, coordination and balance, making it particularly suitable for COPD patients, as observed for elderly people in terms of risk of fall [5]. In addition to the interesting results obtained by СRоок et al. [1] we suggest that physiological and objective anchors be favoured while estimating the MID of these new simple field tests to better characterise their properties.

Relative changes in 1-min STS performance (i.e. change expressed as per cent of baseline) may also be a simple and practical approach for determining the meaningful response of a test (as we usually do to define the bronchodilator response with the change in FEV1 and/or forced vital capacity). This seems appropriate with the STS test, which is less sensitive to baseline variability compared to the 6MWD. In our study, the magnitude of change after pulmonary rehabilitation did not correlate with the initial STS value, while this is the case with the $6 \mathrm{MWD}(\mathrm{r}=-0.002$, nonsignificant for STS, versus $\mathrm{r}=-0.42, \mathrm{p}=0.006$ for 6MWD) (figure 1). We would be interested to know if the authors observed similar results when comparing the changes of STS, 6MWD and feeling thermometer scale. In that case, this would reinforce the interest of STS test over the 6MWT, with the representation of the STS change as per cent of baseline value.

@ERSpublications

The change in the sit-to-stand test after a pulmonary rehabilitation is not influenced by the initial value http://ow.ly/i2CI30eqprD

Cite this article as: Chambellan A, Nusinovici S, Vaidya T, et al. The validation of the sit-to-stand test for COPD patients. Eur Respir J 2017; 50: 1701203 [https://doi.org/10.1183/13993003.01203-2017]. 

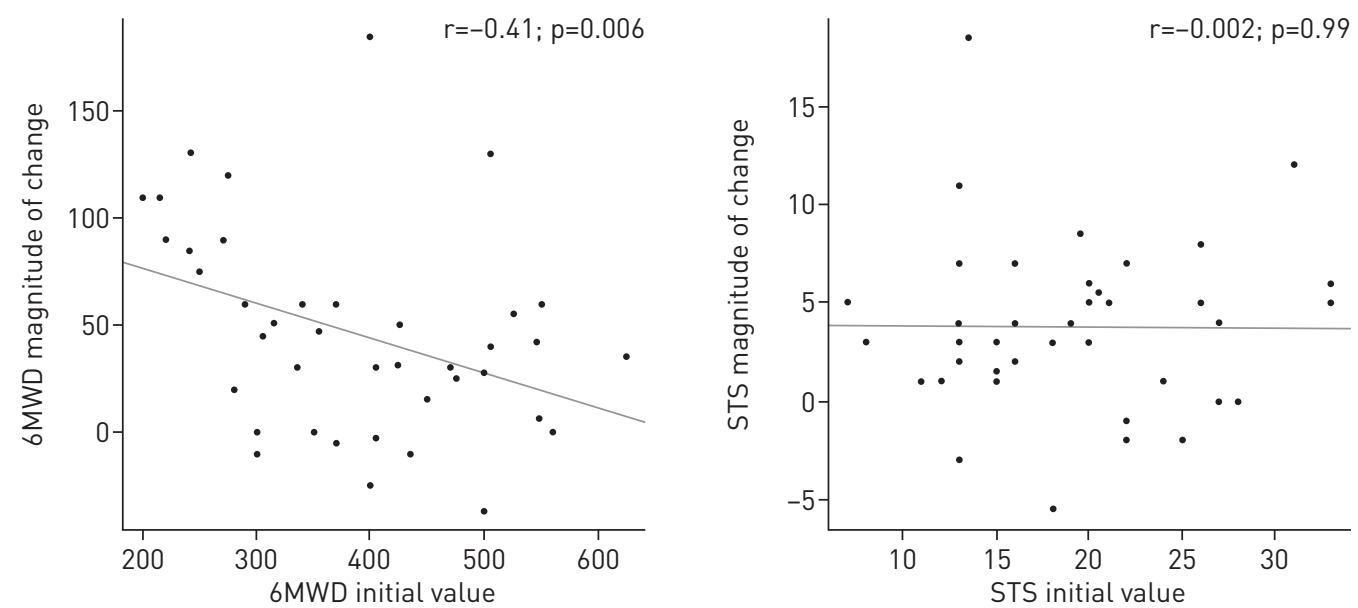

FIGURE 1 Spearman correlation coefficient for the magnitude of change in 6-min walk distance (6MWD) and sit-to-stand (STS) test with their respective initial values.

Whether the STS test will be the future gold standard of home-based monitoring of physical capacity and HRQoL still remains to be confirmed, but it seems to present many advantages that put it in a good position.

Arnaud Chambellan ${ }^{1,2}$, Simon Nusinovici ${ }^{3}$, Trija Vaidya $\odot^{4}$, Pierre-Antoine Gourraud ${ }^{3,5}$ and Claire de Bisschop ${ }^{4}$ ${ }^{1}$ UNIV Nantes, Laboratory "Movement, Interactions, Performance", Nantes, France. ${ }^{2}$ L'Institut du thorax, CHU Nantes, Nantes, France. ${ }^{3}$ Clinique des données, CHU de Nantes, Nantes, France. ${ }^{4}$ Université de Poitiers, Laboratoire MOVE EA 6314, Poitiers, France. ${ }^{5}$ INSERM, CIC 1413, Nantes, France.

Correspondence: Arnaud Chambellan, Explorations Fonctionnelles, Hôpital Laennec, CHU Nantes, Boulevard Monod, 44093 Nantes, cedex 1, France. E-mail: Arnaud.Chambellan@chu-nantes.fr

Received: June 192017 | Accepted: June 192017

Conflict of interest: None declared.

\section{References}

1 Crook S, Büsching G, Schultz K, et al. A multicentre validation of the 1-min sit-to-stand test in patients with COPD. Eur Respir J. 2017; 49: 1601871.

2 Reychler G, Boucard E, Peran L, et al. One minute sit-to-stand test is an alternative to 6MWT to measure functional exercise performance in COPD patients. Clin Respir J 2017; in press [https://doi.org/10.1111/crj.12658].

3 Vaidya T, de Bisschop C, Beaumont M, et al. Is the 1-minute sit-to-stand test a good tool for the evaluation of the impact of pulmonary rehabilitation? Determination of the minimal important difference in COPD. Int J Chron Obstruct Pulmon Dis 2016; 11: 2609-2616.

4 Spruit MA, Singh SJ, Garvey C, et al. An official American Thoracic Society/European Respiratory Society statement: key concepts and advances in pulmonary rehabilitation. Am J Respir Crit Care Med 2013; 188: e13-e64.

5 Vaidya T, Chambellan A, de Bisschop C. Sit-to-stand tests for COPD: A Literature review. Respir Med 2017; 128 $70-77$.

Copyright @ERS 2017

From the authors:

We thank A. Chambellan and colleagues for their interest in our study on the validation of the 1-min sit-to-stand (STS) test in chronic obstructive pulmonary disease (COPD) patients published in the European Respiratory Journal [1]. The authors highlighted some discrepancies between our study results

Cite this article as: Crook S, Puhan and MA, Frei A. The validation of the sit-to-stand test for COPD patients. Eur Respir J 2017; 50: 1701506 [https://doi.org/10.1183/13993003.01506-2017]. 
and the results that they found in a similar study of COPD patients, raised questions over the possible cause of this difference and discuss results from their own study that they believe may provide additional information on the responsiveness of the 1-min STS test.

A. Chambellan and colleagues noted that the correlation between the post-pulmonary rehabilitation change scores in 1-min STS test and 6-min walk test (6MWT) observed in the STAND-UP study ( $\mathrm{r}=$ -0.08) was weak in comparison to the correlation coefficient observed in their own study ( $\mathrm{r}=0.50)$ [2]. They consider whether the discrepancies could be explained by differences in pulmonary rehabilitation programmes or severity of disease in the two populations. However, the patients from their study were similar to the patients of the RIMTCORE study in terms of forced expiratory volume in $1 \mathrm{~s}$ (\% predicted), where the change score correlations were also weak $(r=0.21)$. This suggests that severity of disease may not be a driving factor for the discrepancies. Although there is a moderate to strong cross-sectional correlation between the two tests in all three studies ( $\mathrm{r}=0.57$ to 0.68 ), indicating that the 1 -min STS test is a good measure of functional exercise capacity, the discrepancies in change scores suggest that the two tests reacted to the pulmonary rehabilitation programmes in different ways. This could be attributed to differences between the pulmonary rehabilitation programmes, or simply differences in the manner that the two tests respond to the intervention. However, currently we can only speculate about the reason for the discrepancies seen between the three samples.

The correspondence proposed the use of physiological and objective measures as anchors to estimate the minimal important difference (MID), instead of patient-reported outcomes. While it would be ideal to use an anchor that measures the same construct (functional exercise capacity) as the 1-min STS test if the correlation of the change scores is moderate to high, this was not the case in our study. However, patient-reported outcomes still capture some aspects associated with impaired functional exercise capacity (such as dyspnoea and fatigue) and may be suitable as anchors when the correlation is moderate or high. We believe that our use of combining several different methods to estimate the MID, and not relying exclusively on the anchor-based method with the feeling thermometer, was appropriate to provide a reliable estimate [3]. It should also be noted that it is quite controversial to use physiological measures as anchors since it can be argued that the concept of the MID, by definition reflecting the patient perspective, may not be applicable to physiological measures [4]. Confidence in a specific MID usually evolves over time and is confirmed by additional evidence. Interestingly, the study by A. Chambellan and colleagues where the 6MWT was used as an anchor, along with distributional methods, found the same MID estimate as in our study [2].

While information on the sensitivity of the 1-min STS test to baseline values in comparison to other tests may add additional insights into the test, we do not believe that the analysis of differential baseline effects in the manner proposed by A. Chambellan and colleagues would be appropriate to assess responsiveness of the test due to potential statistical issues that can occur, limiting the inferences that can be made. Oldham [5] already pointed out that even if baseline and follow-up values are completely uncorrelated, the correlation between baseline values and the change scores (follow-up minus baseline) is about -0.7. This of course makes little sense and is due to regression to the mean and mathematical coupling [5-7]. Oldham [5] proposed instead to compare the average of the two values to the difference between the two, as Bland and Altman [8] later also suggested, and summarises this using the Pearson correlation between the mean of the scores, and their difference.

Sarah Crook, Milo A. Puhan and Anja Frei and on behalf of the STAND-UP and RIMTCORE study groups Epidemiology, Biostatistics and Prevention Institute, Dept of Epidemiology, University of Zurich, Zurich, Switzerland.

Correspondence: Sarah Crook, Epidemiology, Biostatistics and Prevention Institute, Dept of Epidemiology, University of Zurich, Zurich, CH-8001, Switzerland. E-mail: sarah.crook@uzh.ch

Received: July 242017 | Accepted: July 252017

Conflict of interest: None declared.

\section{References}

1 Crook S, Büsching G, Schultz K, et al. A multicentre validation of the 1-minute sit-to-stand test in patients with COPD. Eur Respir J 2017; 49: 1601871.

2 Vaidya T, de Bisschop C, Beaumont M, et al. Is the 1-minute sit-to-stand test a good tool for the evaluation of the impact of pulmonary rehabilitation? Determination of the minimal important difference in COPD. Int J COPD 2016; 11: 2609-2616.

3 Revicki D, Hays RD, Cella D, et al. Recommended methods for determining responsiveness and minimally important differences for patient-reported outcomes. J Clin Epidemiol 2008; 61: 102-109.

4 Guyatt G, Montori V, Devereaux P, et al. Patients at the centre: in our practice, and in our use of language. Evid Based Med 2004; 9: 6-7. 
5 Oldham PD. A note on the analysis of repeated measurements of the same subjects. J Chronic Dis 1962; 15: 969-977.

6 Archie JP. Mathematic coupling of data: a common source of error. Ann Surg 1981; 193: 296-303.

$7 \mathrm{Tu}$ YK, Gilthorpe MS. Revisiting the relation between change and initial value: A review and evaluation. Stat Med 2007; 26: 443-457.

8 Bland JM, Altman D. Statistical methods for assessing agreement between two methods of clinical measurement. Lancet 1986; 327: 307-310. 